\title{
A Class of Nearly Sub-Weyl and Sub-Lyra Manifolds
}

\author{
Han Yanling, Peibiao Zhao* \\ (Communicated by Uday Chand De)
}

\begin{abstract}
The authors introduce a nearly sub-Weyl and a nearly sub-Lyra manifold by virtue of a SNS-nonmetric connection(defined below) in nearly sub-Riemannian manifolds. In particular, we show a SR-parallel curve associated with the nearly sub-Lyra connection is actually a minimizer of the horizontal length functional. A geometric characteristic of a SNS-non-metric connection is given in the last section.
\end{abstract}

\section{Introduction}

H.Weyl [12] introduced a generalization of Riemannian geometry in order to formulate a unified field theory. Weyl's theory provides an instructive example of non-Riemannian connection. It is well known that a Weyl connection is actually a torsion-free but non-metric connection, namely the so-called symmetric non-metric connection in transformation's theory. In 1951 G.Lyra [7] suggested a semi-symmetric metric connection and introduced the notion of "gauge", which made it possible to construct a geometrized theory of gravitation and electromagnetism along the lines of Weyl theory.

The study of various connections on Riemannian or non-Riemannian manifolds has been an active field over the past seven decades. In particular, since the formidable papers $[1,3,4,11,13]$ were published in succession, these works stimulate such research fields to present a scene of prosperity, and demonstrate the abnormal importance of this topic. We have made some attempts in this field and obtained some results, see [6, 14].

In order to study sub-Riemannian geometry from the point of theory of transformation, we need to define an analogue of the Levi-Civita connection. Because of no metric defined on the entire tangent bundle, A.Bejauce defined a new sub-Riemannian connection in [2] and discussed the corresponding geometry that is very similar to Riemannian geometry, which motivate us to replace the non-holonomic connection in [6] by this new subRiemannian connection.

In this paper, we, based on the work by A.Bejauce [2], following the ideas of D.K.Sen and J.R.Vanstone in [10], consider the nearly sub-Lyra manifold and the nearly sub-Weyl manifold by virtue of a SNS-nonmetric connection(defined below) in nearly sub-Riemannian manifolds, in contrast to our former papers, a non-holonomic connection(i.e. the projection of Riemannian connection on horizontal distribution) will be replaced by a horizontal sub-Riemannian connection.

The organization of this paper is as follows. In section 2, we will recall and give some necessary notations and terminologies about horizontal sub-Riemannian connection and nearly sub-Riemannian manifolds. Section 3 and Section 4 are concentrated on the sub-Weyl and sub-Lyra's geometries. Section 5 is devoted to the SNSnon-metric connection and its geometric characteristic. An example is given in the last section. 


\section{Preliminaries}

Let $\left(M, V_{0}, g\right)$ be a $n$-dimensional sub-Riemannian manifold, in particular, when $V_{0}=T M$, then $M$ is degenerated into a Riemanian manifold and hence there are no abnormal geodesics(see [8] for details). Without loss of generality, we assume $V_{0} \neq T M$, if $V_{0}$ is strongly bracket generating, then there does not exist non-trivial abnormal geodesics (see [9]). In order to study the sub-Riemannian geometry, we suppose that there exists a complementary distribution $V_{1}$ to $V_{0}$ in the tangent bundle $T M$ of $M$. We note that $V_{1}$ exists on any paracompact sub- Riemannian manifold. Indeed, in this case, there exists a Riemannian metric $g^{*}$ on $M$ and $V_{1}$ is taken as the complementary orthogonal distribution to $V_{0}$ in $T M$, with respect to $g^{*}$. If not stated otherwise, we suppose that $V_{1}$ is an integrable distribution on $M$.

Throughout the paper, we denote by $F(M)$ the set of smooth functions on $M, \Gamma\left(V_{0}\right)$ the $C^{\infty}(M)$-module of smooth sections on $V_{0}$ and $X, Y, Z, \cdots$ the vector fields in $\Gamma(T M), X_{0}$ the projection of $X$ on $V_{0}, X_{1}$ the projection of $X$ on $V_{1}$. The repeated indices with one upper index and one lower index indicates summation over their range. We use the following ranges for indices: $i, j, k, h, \cdots \in\{1, \cdots, \ell\}, \alpha, \beta, \cdots \in\{\ell+1, \cdots, n\}$.

We define a 3-multilinear mapping by

$$
\begin{aligned}
& \Omega: \Gamma\left(V_{0}\right) \times \Gamma\left(V_{0}\right) \times \Gamma\left(V_{1}\right) \rightarrow F(M) \\
& \Omega\left(X_{0}, Y_{0}, Z_{1}\right)=Z_{1} g\left(X_{0}, Y_{0}\right)-g\left(\left[Z_{1}, X_{0}\right]_{0}, Y_{0}\right)-g\left(\left[Z_{1}, Y_{0}\right]_{0}, X_{0}\right),
\end{aligned}
$$

It is easy to check $\Omega$ is a tensor field by a direct computation.

Definition 2.1. We say that a sub-Riemannian manifold $\left(M, V_{0}, g\right)$ is a nearly sub-Riemannian manifold if the tensor field $\Omega$ vanishes identically on $M$.

Theorem 2.1. [2] Given a nearly sub-Riemannian manifold $\left(M, V_{0}, g\right)$, then there exists a unique linear connection $\nabla: \Gamma(T M) \times \Gamma\left(V_{0}\right) \rightarrow \Gamma\left(V_{0}\right)$ satisfying

$$
\begin{gathered}
\left(\nabla_{Z} g\right)\left(X_{0}, Y_{0}\right)=Z\left(g\left(X_{0}, Y_{0}\right)\right)-g\left(\nabla_{Z} X_{0}, Y_{0}\right)-g\left(X_{0}, \nabla_{Z} Y_{0}\right)=0 \\
T\left(X, Y_{0}\right)=\nabla_{X} Y_{0}-\nabla_{Y_{0}} X_{0}-\left[X, Y_{0}\right]_{0}=0 .
\end{gathered}
$$

It is not hard to derive that the connection determined by Equation (2.1) and (2.2) is of the form

$$
\begin{aligned}
2 g\left(\nabla_{X_{0}} Y_{0}, Z_{0}\right) & =X_{0} g\left(Y_{0}, Z_{0}\right)+Y_{0} g\left(Z_{0}, X_{0}\right)-Z_{0} g\left(X_{0}, Y_{0}\right) \\
& +g\left(\left[X_{0}, Y_{0}\right]_{0}, Z_{0}\right)-g\left(\left[Y_{0}, Z_{0}\right]_{0}, X_{0}\right)+g\left(\left[Z_{0}, X_{0}\right]_{0}, Y_{0}\right), \\
\nabla_{Z_{1}} X_{0} & =\left[Z_{1}, X_{0}\right]_{0},
\end{aligned}
$$

Its torsion is defined by

$$
\begin{aligned}
& T: \Gamma(T M) \times \Gamma\left(V_{0}\right) \rightarrow \Gamma\left(V_{0}\right) \\
& T\left(X, Y_{0}\right)=\nabla_{X} Y_{0}-\nabla_{Y_{0}} X_{0}-\left[X, Y_{0}\right]_{0}
\end{aligned}
$$

By Theorem 2.1, we know

$$
T\left(X_{1}, Y_{0}\right)=\nabla_{X_{1}} Y_{0}-\left[X_{1}, Y_{0}\right]_{0}=0
$$

so (2.2) is equivalent to

$$
T\left(X_{0}, Y_{0}\right)=\nabla_{X_{0}} Y_{0}-\nabla_{Y_{0}} X_{0}-\left[X_{0}, Y_{0}\right]_{0}=0 .
$$

Definition 2.2. A linear connection satisfying (2.1) and (2.2)(or (2.3)) is called a horizontal sub-Riemannian connection, in briefly, a HSR-connection.

Next we consider the curvature tensor $R$ of $\nabla$ :

$$
\begin{aligned}
& R: \Gamma(T M) \times \Gamma(T M) \times \Gamma\left(V_{0}\right) \rightarrow \Gamma\left(V_{0}\right) \\
& R\left(X, Y, Z_{0}\right)=\nabla_{X} \nabla_{Y} Z_{0}-\nabla_{X} \nabla_{Y} Z_{0}-\nabla_{[X, Y]} Z_{0},
\end{aligned}
$$

Hereafter we call $R$ the horizontal curvature tensor because of $R\left(X, Y, Z_{0}\right) \in \Gamma\left(V_{0}\right)$. 
Now we consider the local coordinate $\left(x^{i}, x^{\alpha}\right)$, such that $\left(\partial / \partial x^{\ell}, \cdots, \partial / \partial x^{n}\right)$ is a local basis for $V_{1}$ and $V_{0}$ is locally given by the Pfaff system

$$
\delta x^{\alpha}=d x^{\alpha}+A_{i}^{\alpha} d x^{i},
$$

where $A_{i}^{\alpha}$ are smooth functions locally defined on $M$. Thus

$$
e_{i}=\frac{\partial}{\partial x^{i}}-A_{i}^{\alpha} \frac{\partial}{\partial x^{\alpha}},
$$

form a local basis of $V_{0}$. We call $\left(x^{i}, x^{\alpha}\right)$ and $\left(e_{i}, \partial / \partial x^{\alpha}\right)$ an adapted coordinate system and an adapted frame field on $M$ induced by the foliation determined by $V_{1}$. Then by a direct calculation, we obtain

$$
\Omega\left(e_{i}, e_{j}, \frac{\partial}{\partial x^{\alpha}}\right)=\frac{\partial g_{i j}}{\partial x^{\alpha}},
$$

where $g_{i j}=g\left(e_{i}, e_{j}\right)$ is the local component of the Riemannian metric $g$ on $V_{0}$, so the sub-Riemannian manifold $\left(M, V_{0}, g\right)$ is a nearly sub-Riemannian manifold if and only if $\frac{\partial g_{i j}}{\partial x^{\alpha}}=0$, namely, $g_{i j}$ is independent of $x^{\alpha}$. We denote by

$$
\begin{aligned}
& \nabla_{e_{i}} e_{j}=\nabla_{i} e_{j}=\left\{\begin{array}{l}
k \\
i j
\end{array}\right\} e_{k}, \nabla_{\frac{\partial}{\partial x^{\alpha}}} e_{j}=\nabla_{\alpha} e_{j}=\left\{\begin{array}{l}
k \\
\alpha j
\end{array}\right\} e_{k}, R\left(e_{i}, e_{j}, e_{k}\right)=R_{i j k}^{h} e_{h}, \\
& R\left(\frac{\partial}{\partial x^{\alpha}}, e_{j}, e_{k}\right)=R_{\alpha j k}^{h} e_{h}, R\left(\frac{\partial}{\partial x^{\alpha}}, \frac{\partial}{\partial x^{\beta}}, e_{k}\right)=R_{\alpha \beta k}^{h} e_{h},
\end{aligned}
$$

According to Theorem 2.1, we obtain

$$
\left\{\begin{array}{l}
k \\
i j
\end{array}\right\}=\frac{1}{2} g^{k h}\left(\frac{\partial g_{i h}}{\partial x^{j}}+\frac{\partial g_{j h}}{\partial x^{i}}-\frac{\partial g_{i j}}{\partial x^{h}}\right),\left\{\begin{array}{l}
k \\
\alpha j
\end{array}\right\}=0,
$$

and

$$
R_{i j k}^{h}=e_{i}\left(\left\{\begin{array}{c}
h \\
j k
\end{array}\right\}\right)-e_{j}\left(\left\{\begin{array}{c}
h \\
i k
\end{array}\right\}\right)+\left\{\begin{array}{c}
e \\
j k
\end{array}\right\}\left\{\begin{array}{c}
h \\
i e
\end{array}\right\}-\left\{\begin{array}{c}
e \\
i k
\end{array}\right\}\left\{\begin{array}{c}
h \\
j e
\end{array}\right\}, R_{\alpha j k}^{h}=0, R_{\alpha \beta k}^{h}=0 .
$$

Denote by $R_{i j k h}=R_{i j k}^{l} g_{l h}, R_{i k}=R_{i j k h} g^{j h}$, we further have

$$
\left\{\begin{array}{l}
R_{i j k h}=-R_{i j h k}=-R_{j i k h}, \\
R_{i j k h}=R_{k h i j}, \\
R_{i j k h}+R_{j k i h}+R_{k i j h}=0, \\
R_{i j k l, h}+R_{i j l h, k}+R_{i j h k, l}=0,
\end{array}\right.
$$

where $R_{i j k l, h}=\left(\nabla_{h} R\right)\left(e_{i}, e_{j}, e_{k}, e_{l}\right)$ is the covariant derivative of $R$ with respect to $e_{h}$ (see [2]).

\section{A nearly sub-Weyl manifold}

Weyl's idea provides an instructive example of non-Riemannian connections, further G.B.Folland [5] had given a global formulation of Weyl manifolds thereby clarifying considerably many of Weyl's basic ideas. Based on these facts, we, in this subsection, aim at the geometries of the nearly sub-Riemannian manifold equipped with a Weyl structure.

Theorem 3.1. (Existence) Given a nearly sub-Riemannian manifold $\left(M, V_{0}, g\right)$ and a 1-form $\varphi$, then there exists a unique connection $\hat{\nabla}: \Gamma(T M) \times \Gamma\left(V_{0}\right) \rightarrow \Gamma\left(V_{0}\right)$ on $M$ such that

$$
\left\{\begin{array}{l}
\left(\hat{\nabla}_{Z} g\right)\left(X_{0}, Y_{0}\right)=-\varphi(Z) g\left(X_{0}, Y_{0}\right), \\
\hat{T}\left(X, Y_{0}\right)=\hat{\nabla}_{X} Y_{0}-\hat{\nabla}_{Y_{0}} X_{0}-\left[X, Y_{0}\right]_{0}=0 .
\end{array}\right.
$$

Proof. From the second equation of (3.1), one can derive the connection $\hat{\nabla}$ is necessarily of the form,

$$
\hat{\nabla}_{X_{1}} Y_{0}=\left[X_{1}, Y_{0}\right]_{0} .
$$


And (3.1) implies

$$
g\left(\hat{\nabla}_{Z_{0}} X_{0}, Y_{0}\right)=Z_{0}\left(g\left(X_{0}, Y_{0}\right)\right)-g\left(X_{0}, \hat{\nabla}_{Y_{0}} Z_{0}\right)-g\left(X_{0},\left[Z_{0}, Y_{0}\right]_{0}\right)-\varphi\left(X_{0}\right) g\left(Z_{0}, Y_{0}\right),
$$

By cyclic permutation of $X, Y, Z$, one obtains another two equations (3.2') and (3.2"), if we add (3.2') and (3.2"), and subtract (3.2), one gets

$$
\begin{aligned}
2 g\left(\hat{\nabla}_{X_{0}} Y_{0}, Z_{0}\right)= & X_{0}\left(g\left(Y_{0}, Z_{0}\right)\right)+Y_{0}\left(g\left(Z_{0}, X_{0}\right)\right)-Z_{0}\left(g\left(X_{0}, Y_{0}\right)\right) \\
& +g\left(\left[X_{0}, Y_{0}\right]_{0}, Z_{0}\right)-g\left(\left[Y_{0}, Z_{0}\right]_{0}, X_{0}\right)+g\left(\left[Z_{0}, X_{0}\right]_{0}, Y_{0}\right) \\
& +\varphi\left(X_{0}\right) g\left(Z_{0}, Y_{0}\right)+\varphi\left(Y_{0}\right) g\left(Z_{0}, X_{0}\right)-\varphi\left(Z_{0}\right) g\left(X_{0}, Y_{0}\right),
\end{aligned}
$$

Since $g$ is non-degenerate on horizontal distribution $V_{0}$, it defines $\hat{\nabla}_{X_{0}} Y_{0}$ uniquely as follows,

$$
\left\{\begin{array}{l}
\hat{\nabla}_{X_{0}} Y_{0}=\nabla_{X_{0}} Y_{0}+\frac{1}{2}\left(\varphi\left(X_{0}\right) Y_{0}+\varphi\left(Y_{0}\right) X_{0}-g\left(X_{0}, Y_{0}\right) P\right) \\
\hat{\nabla}_{X_{1}} Y_{0}=\left[X_{1}, Y_{0}\right]_{0},
\end{array}\right.
$$

where $P$ is a horizontal vector field defined by $g\left(X_{0}, P\right)=\varphi\left(X_{0}\right)$.

Conversely, the connection defined by (3.3) satisfies necessarily (3.1). Therefore, (3.3) define uniquely the connection.

This completes the proof.

Definition 3.1. A connection is said to be a nearly sub-Weyl connection, or a nearly sub-Weyl transformation from the point of transformation's theory, if it satisfies (3.3). A nearly sub-Riemannian manifold $\left(M, V_{0}, g\right)$ is said to be a nearly sub-Weyl manifold denoted by $\left(M, V_{0}, g, \varphi\right)$, if $M$ admits a nearly sub-Weyl connection.

According to Theorem 3.1, a nearly sub-Weyl connection is uniquely determined by the sub-Riemannian metric $g$ and a 1-form $\varphi$. In our adapted coordinates, the coefficients of nearly sub-Weyl connection are given by

$$
\hat{\Gamma}_{i j}^{k}=\left\{{ }_{i j}^{k}\right\}+\frac{1}{2}\left(\varphi_{i} \delta_{j}^{k}+\varphi_{j} \delta_{i}^{k}-\varphi^{k} g_{i j}\right), \hat{\Gamma}_{\alpha j}^{k}=0,
$$

where $\varphi_{i}=\varphi\left(e_{i}\right)$ and $\varphi^{k}=\varphi_{i} g^{i k}$. Such a connection is called a nearly Weyl transformation from the point of transformation theory.

Definition 3.2. An absolutely continuous curve $\gamma: x^{a}=x^{a}(t)$ is said to be a horizontal invariant curve if it satisfies $\nabla_{\dot{\gamma}} \dot{\gamma}_{0}=\alpha(t) \dot{\gamma}_{0}$, where $t$ is any parameter and $\alpha(t)$ is a function related to $\gamma$. In particular, $\gamma$ is called a sub-Riemannian parallel curve(in short, a SR-parallel curve) if $\nabla_{\dot{\gamma}} \dot{\gamma}_{0}=0$.

Remark 3.1. It is obvious that a SR-parallel curve is necessarily a horizontal invariant curve. In contrast to the path that Jiao and Zhao defined in [14], the horizontal invariant curve is not necessarily a horizontal curve(i.e. $\dot{\gamma} \in V_{0}$ ). On the other hand, this kind of SR-parallel curve is exactly a Riemannian geodesic when the horizontal bundle is the whole tangent bundle.

Remark 3.2. The image curve of a a horizontal invariant curve with respect to the HSR-connection is not a horizontal invariant curve with respect to the nearly sub-Weyl connection any more under a nearly sub-Weyl transformation.

In fact, for an absolutely continuous curve $\gamma: x^{a}=x^{a}(t)$ with tangent vector

$$
\dot{\gamma}=\frac{d x^{i}}{d t} \frac{\partial}{\partial x^{i}}+\frac{d x^{\alpha}}{d t} \frac{\partial}{\partial x^{\alpha}}=\frac{d x^{i}}{d t} e_{i}+\left(A_{i}^{\alpha} \frac{d x^{i}}{d t}+\frac{d x^{\alpha}}{d t}\right) \frac{\partial}{\partial x^{\alpha}},
$$

If $\gamma: x^{a}=x^{a}(t)$ is a horizontal invariant curve with respect to the HSR-connection, then it satisfies the equation $\nabla_{\dot{\gamma}} \dot{\gamma}_{0}=\alpha(t) \dot{\gamma}_{0}$, namely

$$
\nabla_{\dot{\gamma}} \dot{\gamma}_{0}=\nabla_{\dot{\gamma}_{0}} \dot{\gamma}_{0}+\nabla_{\dot{\gamma}_{1}} \dot{\gamma}_{0}=\alpha(t) \dot{\gamma}_{0},
$$

Next we compute the second term $\nabla_{\dot{\gamma}_{1}} \dot{\gamma}_{0}$. According to the definition of HSR-connection, one has

$$
\begin{aligned}
\nabla_{\dot{\gamma}_{1}} \dot{\gamma}_{0}= & {\left[\dot{\gamma}_{1}, \dot{\gamma}_{0}\right]_{0} } \\
= & {\left[\left(A_{i}^{\alpha} \frac{d x^{i}}{d t}+\frac{d x^{\alpha}}{d t}\right) \frac{\partial}{\partial x^{\alpha}}, \frac{d x^{i}}{d t} e_{i}\right]_{0} } \\
= & \left\{\left(A_{i}^{\alpha} \frac{d x^{i}}{d t}+\frac{d x^{\alpha}}{d t}\right) \frac{\partial}{\partial x^{\alpha}}\left(\frac{d x^{i}}{d t}\right) e_{i}-\frac{d x^{i}}{d t} e_{i}\left(A_{i}^{\alpha} \frac{d x^{i}}{d t}+\frac{d x^{\alpha}}{d t}\right) \frac{\partial}{\partial x^{\alpha}}\right. \\
& \left.+\left(A_{i}^{\alpha} \frac{d x^{i}}{d t}+\frac{d x^{\alpha}}{d t}\right) \frac{d x^{i}}{d t} \frac{\partial}{\partial x^{\alpha}}\left(A_{i}^{\beta}\right) \frac{\partial}{\partial x^{\beta}}\right\}_{0} \\
= & 0 .
\end{aligned}
$$


in the adapted frame system, we derive the equations of horizontal invariant curve with respect to the HSRconnection

$$
\frac{d^{2} x^{k}}{d t^{2}}+\left\{{ }_{i j}^{k}\right\} \frac{d x^{i}}{d t} \frac{d x^{j}}{d t}=\alpha(t) \frac{d x^{k}}{d t} .
$$

Now we substitute (3.4) into Equation (3.5), then one gets

$$
\frac{d^{2} x^{k}}{d t^{2}}+\hat{\Gamma}_{i j}^{k} \frac{d x^{i}}{d t} \frac{d x^{j}}{d t}+\frac{1}{2} \varphi^{k} g_{i j} \frac{d x^{i}}{d t} \frac{d x^{j}}{d t}=\beta(t) \frac{d x^{k}}{d t} .
$$

Then, $\gamma$ is not a horizontal invariant curve with respect to the nearly sub-Weyl connection. Hence the proof is finished.

\section{A nearly sub-Lyra manifold}

G.Lyra [7] suggested a modification of Riemannian geometry which bears a remarkable resemblance to Weyl's geometry. According to Lyra's ideas, the vector $\overrightarrow{P P^{\prime}}$ between two neighbouring points $P\left(x^{a}\right)$ and $P^{\prime}\left(x^{a}+d x^{a}\right)$ has the components $\xi^{a}=x^{0} d x^{a}$, where $x^{0}\left(x^{i}\right)$ is a gauge function. The adapted coordinate system $\left(x^{a}\right)$ together with the gauge $x^{0}$ form a local adapted reference system $\left(x^{0} ; x^{a}\right)$ which induces a local natural basis $\left\{\tilde{e}_{i}=\frac{1}{x^{0}} \frac{\partial}{\partial x^{2}}\right\}$. We apply this idea into our sub-Riemannian geometry. We assume $\tilde{g}$ be a natural extension of sub-Riemannian metric $g$ such that $V_{0}=\operatorname{span}\left\{\frac{\partial}{\partial x^{i}}: i=1,2, \cdots, \ell\right\}, V_{1}=\operatorname{span}\left\{\frac{\partial}{\partial x^{\alpha}}: \alpha=\ell+1, \cdots, n\right\}$, where $x_{0}$ is independent of $x^{\alpha}$, then $\left\{\tilde{e}_{i}=\frac{1}{x^{0}} \frac{\partial}{\partial x^{i}}\right\}$ form the local reference vector fields of $V_{0}$, and the Lie bracket of any two vector fields in local reference vector fields,

$$
\left[\tilde{e}_{i}, \tilde{e}_{j}\right]=\frac{1}{2}\left(\phi_{i}^{0} \delta_{j}^{k}-\phi_{j}^{0} \delta_{i}^{k}\right) \tilde{e}_{k},
$$

where $\phi_{i}^{0}=2 \frac{\partial}{\partial x^{i}}\left(\frac{1}{x^{0}}\right)$. If we denote the components of the sub-Riemannian metric tensor $g$, in a local reference system, by

then the metric form is

$$
g_{i j}=g\left(\tilde{e}_{i}, \tilde{e}_{j}\right)
$$

$$
d s^{2}=\left(x^{0}\right)^{2} g_{i j} d x^{i} d x^{j},
$$

Theorem 4.1. (Existence) Given a nearly sub-Riemannian manifold $\left(M, V_{0}, g\right)$ and a 1-form $\phi$ with $\phi\left(X_{1}\right)=0$, then Then there exists a unique connection $\tilde{\nabla}: \Gamma(T M) \times \Gamma\left(V_{0}\right) \rightarrow \Gamma\left(V_{0}\right)$ on $M$ such that

$$
\left\{\begin{array}{l}
\left(\tilde{\nabla}_{Z} g\right)\left(X_{0}, Y_{0}\right)=0, \\
\tilde{T}\left(X, Y_{0}\right)=\tilde{\nabla}_{X} Y_{0}-\tilde{\nabla}_{Y_{0}} X_{0}-\left[X, Y_{0}\right]_{0}=\frac{1}{2}\left(\phi\left(Y_{0}\right) X_{0}-\phi(X) Y_{0}\right) .
\end{array}\right.
$$

Proof. By the same method as Theorem 3.1, one can obtain an unique connection determined by Equations $(4.1)$,

$$
\left\{\begin{array}{l}
\tilde{\nabla}_{X_{0}} Y_{0}=\nabla_{X_{0}} Y_{0}+\frac{1}{2}\left(\phi\left(Y_{0}\right) X_{0}-g\left(X_{0}, Y_{0}\right) Q\right), \\
\tilde{\nabla}_{X_{1}} Y_{0}=\left[X_{1}, Y_{0}\right]_{0} .
\end{array}\right.
$$

where $Q$ is a horizontal vector field defined by $g\left(X_{0}, Q\right)=\phi\left(X_{0}\right)$.

Definition 4.1. A connection is called a nearly sub-Lyra connection if it satisfies (4.2), or a nearly sub-Lyra transformation from the point of transformation's theory. A nearly sub-Riemannian manifold $\left(M, V_{0}, g\right)$ is said to be a nearly sub-Lyra manifold denoted by $\left(M, V_{0}, g, \phi\right)$, if $M$ admits a nearly sub-Lyra connection.

Theorem 4.1 implies, a nearly sub-Riemannian manifold is turned out to be a nearly sub-Lyra manifold under a nearly sub-Lyra transformation.

For an absolutely continuous curve $\gamma: x^{a}=x^{a}(t), t \in[0,1]$, we define the horizontal length of $\gamma$ by

$$
L_{0}(\gamma)=\int_{0}^{1} \sqrt{g\left(\dot{\gamma}_{0}, \dot{\gamma}_{0}\right)} d t
$$

and

$$
d_{0}(p, q)=\inf \left\{L_{0}(\gamma): \gamma \text { is an absolutely curve with } \gamma(0)=p, \gamma(1)=q\right\},
$$

A horizontal length minimizer is a curve that realizes the distance $d_{0}(p, q)$. 
Theorem 4.2. Let $\left(M, V_{0}, g, \phi\right)$ be a nearly sub-Lyra manifold, then a SR-parallel curve is actually a minimizer of the horizontal length functional.

Proof. Now we take $X_{0}=\tilde{e}_{i}, Y_{0}=\tilde{e}_{j}, Z_{0}=\tilde{e}_{k}$ in Equation (4.2) and denote the coefficients by $\tilde{\nabla}_{\tilde{e}_{i}} \tilde{e}_{j}=\tilde{\Gamma}_{i j}^{k} \tilde{e}_{k}$, we then arrive at

$$
\tilde{\Gamma}_{i j}^{k}=\frac{1}{x^{0}}\left\{\begin{array}{l}
k \\
i j
\end{array}\right\}+\frac{1}{2}\left(\phi_{i} \delta_{j}^{k}-\phi^{k} g_{i j}\right), \tilde{\Gamma}_{\alpha j}^{k}=0,
$$

where $\phi_{i}=\phi\left(\tilde{e}_{i}\right)+\phi_{i}^{0}, \phi^{k}=\phi_{i} g^{i k}$.

Since a horizontal length minimizer is defined by the extremal curves of the problem in the calculus of variations:

$$
\delta\left(\int_{0}^{1} d s\right)=\delta\left(\int_{0}^{1} \sqrt{\left(x^{0}\right)^{2} g_{i j} \frac{d x^{i}}{d t} \frac{d x^{j}}{d t}} d t\right)=0,
$$

where $s$ is the arc-length and $t$ is an arbitrary parameter, the Lagrangian

$$
L\left(x^{k}, \dot{x}^{k}, t\right)=\sqrt{\left(x^{0}\right)^{2} g_{i j} \frac{d x^{i}}{d t} \frac{d x^{j}}{d t}}=\frac{d s}{d t},
$$

then

$$
\begin{aligned}
\frac{\partial L}{\partial x^{k}} & =\frac{1}{2} \frac{\partial}{\partial x^{k}}\left\{\left(x^{0}\right)^{2} g_{i j}\right\} \frac{d x^{i}}{d t} \frac{d x^{j}}{d s}, \\
\frac{d}{d t}\left(\frac{\partial L}{\partial \dot{x}^{k}}\right) & =\left\{\frac{\partial}{\partial x^{i}}\left[\left(x^{0}\right)^{2} g_{k j}\right] \frac{d x^{i}}{d s} \frac{d x^{j}}{d s}+\left(x^{0}\right)^{2} g_{k j} \frac{d^{2} x^{j}}{d s^{2}}\right\} \frac{d s}{d t} .
\end{aligned}
$$

and hence the Euler-Lagrange equations are equivalent to

$$
\begin{aligned}
\frac{d}{d t}\left(\frac{\partial L}{\partial \dot{x}^{k}}\right)-\frac{\partial L}{\partial x^{k}}=0 \Leftrightarrow & \frac{\partial}{\partial x^{i}}\left[\left(x^{0}\right)^{2} g_{k j}\right] \frac{d x^{i}}{d s} \frac{d x^{j}}{d s}+\left(x^{0}\right)^{2} g_{k j} \frac{d^{2} x^{j}}{d s^{2}}=\frac{1}{2} \frac{\partial}{\partial x^{k}}\left\{\left(x^{0}\right)^{2} g_{i j}\right\} \frac{d x^{i}}{d s} \frac{d x^{j}}{d s} \\
\Leftrightarrow & \left(x^{0}\right)^{2} g_{k j} \frac{d^{2} x^{j}}{d s^{2}}+\left(2 x^{0} \frac{\partial x^{0}}{\partial x^{i}} g_{k j}+\left(x^{0}\right)^{2} \frac{\partial g_{k j}}{\partial x^{i}}\right) \frac{d x^{i}}{d s} \frac{d x^{j}}{d s} \\
& =x^{0} \frac{\partial x^{0}}{\partial x^{k}} g_{i j} \frac{d x^{i}}{d s} \frac{d x^{j}}{d s}+\frac{1}{2}\left(x^{0}\right)^{2} \frac{\partial g_{i j}}{\partial x^{k}} \frac{d x^{i}}{d s} \frac{d x^{j}}{d s} \\
\Leftrightarrow & \left(x^{0}\right)^{2} g_{k j} \frac{d^{2} x^{j}}{d s^{2}}+2 x^{0} \frac{\partial x^{0}}{\partial x^{i}} g_{k j} \frac{d x^{i}}{d s} \frac{d x^{j}}{d s}+\frac{1}{2}\left(x^{0}\right)^{2}\left(\frac{\partial g_{k j}}{\partial x^{i}}+\frac{\partial g_{k i}}{\partial x^{j}}\right) \frac{d x^{i}}{d s} \frac{d x^{j}}{d s} \\
& =x^{0} \frac{\partial x^{0}}{\partial x^{k}} g_{i j} \frac{d x^{i}}{d s} \frac{d x^{j}}{d s}+\frac{1}{2}\left(x^{0}\right)^{2} \frac{\partial g_{i j}}{\partial x^{k}} \frac{d x^{i}}{d s} \frac{d x^{j}}{d s} \\
\Leftrightarrow & g_{k j} \frac{d^{2} x^{j}}{d s^{2}}+\frac{1}{x^{0}}\left\{2 \frac{\partial x^{0}}{\partial x^{i}} g_{k j}-\frac{\partial x^{0}}{\partial x^{k}} g_{i j}\right\} \frac{d x^{i}}{d s} \frac{d x^{j}}{d s} \\
& +\frac{1}{2}\left(\frac{\partial g_{k j}}{\partial x^{i}}+\frac{\partial g_{k i}}{\partial x^{j}}-\frac{\partial g_{i j}}{\partial x^{k}}\right) \frac{d x^{i}}{d s} \frac{d x^{j}}{d s}=0 \\
\Leftrightarrow & \frac{d^{2} x^{l}}{d s^{2}}+\frac{1}{x^{0}}\left\{2 \frac{\partial x^{0}}{\partial x^{i}} g_{k j}-\frac{\partial x^{0}}{\partial x^{k}} g_{i j}\right\} \frac{d x^{i}}{d s} \frac{d x^{j}}{d s} g^{k l}+\{l i j\} \frac{d x^{i}}{d s} \frac{d x^{j}}{d s}=0 \\
\Leftrightarrow & \frac{d^{2} x^{l}}{d s^{2}}+x^{0}\left\{-\frac{\partial}{\partial x^{i}}\left(\frac{1}{x^{0}}\right) \delta_{j}^{l}-\frac{\partial}{\partial x^{j}}\left(\frac{1}{x^{0}}\right) \delta_{i}^{l}+\frac{\partial}{\partial x^{k}}\left(\frac{1}{x^{0}}\right) g_{i j} g^{k l}\right\} \frac{d x^{i}}{d s} \frac{d x^{j}}{d s} \\
& +\left\{{ }_{i j}^{l}\right\} \frac{d x^{i}}{d s} \frac{d x^{j}}{d s}=0 \\
\Leftrightarrow & \frac{d^{2} x^{k}}{d s^{2}}+\left\{{ }_{i j}^{k}\right\} \frac{d x^{i}}{d s} \frac{d x^{j}}{d s}+\frac{x^{0}}{2}\left(\phi_{i}^{0} \delta_{j}^{k}+\phi_{j}^{0} \delta_{i}^{k}-\left(\phi^{0}\right)^{k} g_{i j}\right) \frac{d x^{i}}{d s} \frac{d x^{j}}{d s}=0 .
\end{aligned}
$$

In particular, if we choose the normal gauge $x^{0}=1$, then we have

$$
\frac{d^{2} x^{k}}{d s^{2}}+\left\{{ }_{i j}^{k}\right\} \frac{d x^{i}}{d s} \frac{d x^{j}}{d s}=0
$$

This completes the proof. 
Remark 4.1. Let $\left(M, V_{0}, g, \phi\right)$ be a nearly sub-Lyra manifold, if $\gamma(s)$ whose horizontal tangent vector field is $\dot{\gamma}_{0}=x^{0} \frac{d x^{i}}{d s} \tilde{e}_{i}$ is a SR-parallel curve with respect to the nearly sub-Lyra connection, then it satisfies $\tilde{\nabla}_{\dot{\gamma}} \dot{\gamma}_{0}=0$, i.e.

$$
\frac{d^{2} x^{k}}{d s}+\left\{{ }_{i j}^{k}\right\} \frac{d x^{i}}{d s} \frac{d x^{j}}{d s}+\frac{x^{0}}{2}\left(\phi_{i} \delta_{j}^{k}+\phi_{j} \delta_{i}^{k}-\phi^{k} g_{i j}\right) \frac{d x^{i}}{d s} \frac{d x^{j}}{d s}=\frac{x^{0}}{2}\left(\phi_{i}-\phi_{i}^{0}\right) \frac{d x^{i}}{d s} \frac{d x^{k}}{d s} .
$$

where $s$ is arc-length parameter. By comparing Equation(4.4) with Equation(4.5), one obtains that a SR-parallel curve with respect to the nearly sub-Lyra connection does not coincide with the horizontal length minimizer associated with the metric.

\section{A geometric characterization of a SNS-non-metric connection}

In this subsection, we will considered a class of non-symmetric connection, in briefly, a SNS-non-metric connection, and give a geometric property of a SNS-non-metric connection.

Definition 5.1. Let $\left(M, V_{0}, g\right)$ be a nearly sub-Riemannian manifold, a linear connection $D: \Gamma(T M) \times \Gamma\left(V_{0}\right) \rightarrow$ $\Gamma\left(V_{0}\right)$ is said to be a semi-symmetric non-metric connection, in briefly, a SNS-non-metric connection, if it satisfies

$$
\left\{\begin{array}{l}
\left(D_{Z} g\right)\left(X_{0}, Y_{0}\right)=-\pi\left(X_{0}\right) g\left(Y_{0}, Z_{0}\right)-\pi\left(Y_{0}\right) g\left(X_{0}, Z_{0}\right), \\
T_{D}\left(X, Y_{0}\right)=\pi\left(Y_{0}\right) X_{0}-\pi(X) Y_{0} .
\end{array}\right.
$$

where $\pi$ is a 1 -form.

One can derive that Equations (5.1) determined uniquely a SNS-non-metric connection,

$$
\begin{aligned}
2 g\left(D_{X_{0}} Y_{0}, Z_{0}\right) & =X_{0}\left(g\left(Y_{0}, Z_{0}\right)\right)+Y_{0}\left(g\left(Z_{0}, X_{0}\right)\right)-Z_{0}\left(g\left(X_{0}, Y_{0}\right)\right)+g\left(\left[X_{0}, Y_{0}\right]_{0}, Z_{0}\right) \\
& =-g\left(\left[Y_{0}, Z_{0}\right]_{0}, X_{0}\right)+g\left(\left[Z_{0}, X_{0}\right]_{0}, Y_{0}\right)+2 \pi\left(Y_{0}\right) g\left(X_{0}, Z_{0}\right), \\
D_{X_{1}} Y_{0} & =\left[X_{1}, Y_{0}\right]_{0},
\end{aligned}
$$

namely,

$$
D_{X_{0}} Y_{0}=\nabla_{X_{0}} Y_{0}+\pi\left(Y_{0}\right) X_{0}, D_{X_{1}} Y_{0}=\left[X_{1}, Y_{0}\right]_{0} .
$$

in our adapted frame system, it can be rewritten by

$$
D_{i j}^{k}=\left\{\begin{array}{l}
k \\
i j
\end{array}\right\}+\pi_{j} \delta_{i}^{k}, D_{\alpha j}^{k}=0 .
$$

Definition 5.2. For two classes of SNS-non-metric connection $D_{1}$ and $D_{2}$, let $\bar{\Gamma}_{1}$ and $\bar{\Gamma}_{2}$ be their symmetrization of connection coefficients, if the horizontal invariant curve associated with $\bar{D}_{1}$ corresponds always to that associated with $\bar{D}_{2}$, then we say $D_{1}$ is a SR-projective transformation of $D_{2}$ (or, $D_{2}$ is a SR-projective transformation of $D_{1}$ ).

Theorem 5.1. The SNS-non-metric connection (5.2) is essentially a SR-projective transformation.

Proof. We denote the symmetrization of connection coefficients of (5.2) by $\bar{D}_{i j}^{k}$, then

$$
\bar{D}_{i j}^{k}=\frac{D_{i j}^{k}+D_{j i}^{k}}{2}=\left\{\begin{array}{l}
k \\
i j
\end{array}\right\}+\frac{1}{2}\left(\pi_{i} \delta_{j}^{k}+\pi_{j} \delta_{i}^{k}\right)
$$

If $\gamma: x^{a}=x^{a}(t)$ is a horizontal invariant curve associated with the HSR-connection, then it satisfies Equations (5.2), one can obtain by substituting Equations (5.4) into Equations (5.2),

$$
\frac{d^{2} x^{k}}{d t^{2}}+\bar{D}_{i j}^{k} \frac{d x^{i}}{d t} \frac{d x^{j}}{d t}=\beta(t) \frac{d x^{k}}{d t} \text {. }
$$

where $\beta(t)=\alpha(t)+\pi_{i} \frac{d x^{i}}{d t}$.

The converse statement is also true by simple computation, which means a horizontal invariant curve associated with the SR-connection corresponds that with respect to the SNS-non-metric connection, and hence the proof is finished. 
Theorem 5.2. A connection transformation between a nearly sub-Weyl manifold and a nearly sub-Lyra manifold keeps the horizontal invariant curves unchanged.

Proof. In a local reference system with the normal gauge $x^{0}=1$, by comparing the nearly sub-Weyl connection (3.4) with the nearly sub-Lyra connection (4.3), one has

$$
\tilde{\Gamma}_{i j}^{k}=\hat{\Gamma}_{i j}^{k}-\frac{1}{2} \delta_{i}^{k} \varphi_{j}
$$

if we choose the 1-form $\phi$ in the nearly sub-Lyra connection (4.3) is exactly the 1-form $\varphi$ in the nearly sub-Weyl connection (3.4). Therefore the proof follows from Theorem 5.1.

At the end of this paper, we give a decomposition of a SNS-non-metric connection.

Theorem 5.3. A SNS-non-metric connection always decompose into a nearly sub-Weyl connection and a nearly sub-Lyra connection.

Proof. In a local reference system with the normal gauge $x^{0}=1$, by comparing the nearly sub-Weyl connection (3.4) and the nearly sub-Lyra connection (4.3) with the SNS-non-metric connection (5.3), one has

$$
\begin{aligned}
D_{i j}^{k} & =\left\{\begin{array}{l}
k \\
i j
\end{array}\right\}+\pi_{j} \delta_{i}^{k} \\
& =\left[\frac{1}{2}\left\{\begin{array}{c}
k \\
i j
\end{array}\right\}+\left(\pi_{j} \delta_{i}^{k}+\pi_{i} \delta_{j}^{k}-\pi^{k} g_{i j}\right)\right]+\left[\frac{1}{2}\left\{\begin{array}{l}
k \\
i j
\end{array}\right\}+\left(-\pi_{i} \delta_{j}^{k}+\pi^{k} g_{i j}\right)\right] \\
& =\frac{1}{2}\left[\left\{\begin{array}{l}
k \\
i j
\end{array}\right\}+\frac{1}{2}\left(4 \pi_{j} \delta_{i}^{k}+4 \pi_{i} \delta_{j}^{k}-4 \pi^{k} g_{i j}\right)\right]+\frac{1}{2}\left[\left\{{ }_{i j}^{k}\right\}+\frac{1}{2}\left(\left(-2 \pi_{i}\right) \delta_{j}^{k}-\left(-2 \pi^{k}\right) g_{i j}\right)\right] \\
& =\frac{1}{2} \hat{\Gamma}_{i j}^{k}+\frac{1}{2} \tilde{\Gamma}_{i j}^{k} .
\end{aligned}
$$

This finishes the proof.

\section{Examples}

Example 6.1. (Almost contact metric manifold)

Let $M$ be a $(2 n+1)$-dimensional almost contact manifold endowed with an almost contact strucuture $(\varphi, \xi, \eta)$, where $\varphi$ is a $(1,1)$-tensor field, $\xi$ is a vector field and $\eta$ is a 1 -form such that

$$
\varphi^{2}=-I+\eta \otimes \xi, \eta(\xi)=1 .
$$

If the Riemannian metric $g$ satisfies

$$
g(X, \varphi Y)=-g(\varphi X, Y), g(X, \xi)=\eta(\xi) .
$$

then $(\varphi, \xi, \eta, g)$ is called an almost contact metric structure and $M$ is called an almost contact metric manifold. Now we define a linear connection on such manifold

$$
\tilde{\nabla}_{X} Y=\nabla_{X} Y+\eta(Y) X
$$

where $\nabla$ is the levi-Civita connection associated with Riemannian metric $g$, then we obtain

$$
T(X, Y)=\eta(Y) X-\eta(X) Y, \alpha=-\eta \otimes \eta .
$$

which shows that $\tilde{\nabla}$ is a semi-symmetric non-metric connection.

\section{Acknowledgments}

The second author would like to thank Professor H. Z. Li for his encouragement and help! 


\section{References}

[1] Agache, N. S. and Chafle, M. R., A semi-symmetric non-metric connection on a Riemannian manifold. Indian J. Pure Appl. Math., 23(1992), no. 6, 399-409.

[2] Bejancu, A., Curvature in sub-Riemannian geometry, J. Math. Phys., 53, 023513, (2012), DOI :10.1063/1.3684957

[3] De, U. C. and Biswas, S. C., On a type of semi-symmetric non-metric connection on a Riemannian manifold. Istanbul Univ. Mat. Derg., 55/56(1996/1997), 237-243.

[4] De, U. C. and Kamilya, D., On a type of semi-symmetric non-metric connection on a Riemannian manifold. J. Indian Inst. Sci., 75(1995), 707-710.

[5] Folland, G. B., Weyl manifolds. J. Diff. Geom., 4(1970), 145-153.

[6] Han, Y. L., Fu, F. Y. and Zhao, P. B., On semi-symmetric metric connection in sub-Riemannian manifold. Tamkang Journal of Mathematics, 47(2016), no. 4, 373-384.

[7] Lyra, G., U"ber eine modifikation der riemannschen Geometrie. Math. Z., 54(1951), 52-64.

[8] Montgomery, R., Abnormal minimizers. SIAM J. Control Optim., 32(1994), no. 6, 1605-1620.

[9] Montgomery, R., A Tour of Subriemannian geometries, Their Geodesics and Applications. Math. Surv. and Monographs, 91, AMS, 2002.

[10] Sen, D. K. and Vanstone, J. R., On Weyl and Lyra manifolds. J. Math. Phys., 13(1972), 990-993.

[11] Tripathi, M. M. and Kakar, N., On a semi-symmetric non-metric connection in a Kenmotsu manifold. Bull. Cal. Math. Soc., 16(2001), no. 4, 323-330.

[12] Weyl, H., Gravitation und Elektrizltdt, S.-B. Preuss. Akad. Wiss. Berlin, p. 465. Translated in The principle of relativity, Dover Books, New York, 1918.

[13] Yano, K., On semi-symmetric metric connection. Rev. Roum. Math. Pureset Appl., 15(1970), $1579-1586$.

[14] Zhao, P. B. and Jiao, L., Conformal transformations on Carnot Caratheodory spaces. Nihonkal Mathematical Journal, 17(2006), no. 2, 167-185.

\section{Affiliations}

YANLING HAN

AdDress: Dept. of Applied Mathematics, Nanjing University of Science and Technology, Nanjing 210094, P. R. China;

School of Science, Qilu University of Technology, Jinan 250353, P. R. China

E-MAIL: hanyanling1979@163.com

PEIBIAO ZHAO

AdDress: Dept. of Applied Mathematics, Nanjing University of Science and Technology, Nanjing 210094, P. R. China.

E-MAIL: pbzhao@njust.edu.cn 